\title{
A POSSIBLE SAR ARC ENERGIZATION SOURCE: PRECIPITATING ELECTRONS
}

\author{
D. W. Slater, ${ }^{*}$ C. Gurgiolo, ${ }^{* *}$ J. U. Kozyra, ${ }^{* * *}$ \\ E. W. Kleckner* and J. D. Winningham** \\ ${ }^{*}$ Earth Sciences Department, Pacific Northwest Laboratory, \\ Richland, WA, U.S.A. \\ **Department of Space Sciences, Southwest Research Institute, \\ San Antonio, TX, U.S.A. \\ ***Space Physics Research Laboratory, University of Michigan, \\ Ann Arbor, MI, U.S.A.
}

\section{ABSTRACT}

Coincident measurements by ground-based photometers and satellite-borne electron sensors have shown the association of precipitating electrons and Stable Auroral Red Arcs at midlatitudes. Modeling of these events has suggested that, within the constraints imposed by uncertainties of the electron energy spectrum, the electron influx carries sufficient energy to establish ionospheric temperatures required to power the arcs.

\section{INTRODUCTION}

Photometrically, Stable Auroral Red (SAR) arcs are distinct from the more easily detected auroral emissions resulting from energetic particle precipitation because of their stability (both spatial and intensity), monochromatic emission at $6300 \AA$, and typical subauroral latitude. Although the spectral purity excludes the possibility of significant fluxes of energetic particles into the body of the SAR arc, the presence of lower energy particles $(E<10 \mathrm{eV}$ ) is not necessari7y precluded. A number of investigations have attempted to identify such low energy fluxes. Using measurements from the OGO-4 spacecraft, Chandra et al. $/ 1 /$ reported no change of the integrated flux of suprathermal electrons ( $E>5 \mathrm{eV}$ ) during passage at $900 \mathrm{~km}$ altitude over a SAR arc which occurred on 29 September 1967. Nagy et a 1. /2/ using 0G0-6 measurements of integrated suprathermal electron fluxes ( $\mathrm{E}>10 \mathrm{eV}$ ) at $1000 \mathrm{~km}$ altitude over SAR arcs which occurred during 12 JuTy 1969 and 15 July 1969 have reported that no enhancements of electron fluxes were identifiable. Likewise, Maier et al. 13/ used the soft particle spectrometer ( $5 \mathrm{eV}$ to $15 \mathrm{keV}$ ) carried onboard the ISIS-II spacecraft to analyze electron fluxes above a SAR arc feature during the 18 December 1971 geomagnetic storm, again yielding negative results.

A1ternatively, Prasad et al. /4/ correlated measurements by 0G0-6 of increased integrated suprathermal electron fluxes ( $>10 \mathrm{eV}$ ) with the location of a SAR arc on 27 January 1971. A feature described by Shepherd et al. /5/ as an "unusual" SAR arc was detected from the ISIS-II spacecraft on 4 August $1 \overline{972}$. Measurements from $1400 \mathrm{~km}$ altitude identified increased fluxes of low energy electrons, although further study led to the conclusion that there was no evidence of soft electron precipitation into "norma?" SAR arcs. Final7y, Gurgiolo et a?. $/ 6 /$ recently noted a nearly one-to-one correspondence between precipitating electrons $(\mathrm{E}<8 \mathrm{eV}$ ) and $6300 \AA$ emissions identified as a SAR arc during a single passage of the Dynamics ExpTorer-2 (DE-2) spacecraft.

It is from this foundation of apparently conflicting results that a comprehensive search was conducted for coincident measurements of optical and plasma parameters within, or conjugate to, identifiable SAR arc features. Specifically, this search involved the ground-based mobile automatic scanning photometer (MASP) network operated by the Pacific Northwest Laboratory and the low altitude plasma instrument (LAPI) onboard the DE-2 spacecraft. Following the identification of these coincident data, a series of modeling calculations have been carried out to examine the relationship between observed precipitating electron fluxes and the resultant optical signatures.

\section{OBSERVATIONS}

Briefly, the MASP units are programmed to routinely obtain nighttime atmospheric emission measurements across North America during the performance of sequenced altazimuthal scans. Spectral discrimination is provided by narrow bandwidth interference filters. Each individual MASP is capable of monitoring a circular region with radius of approximately $1200 \mathrm{~km}$. 
The major criterion used for identification of SAR arcs in this paper is the enhancement of monochromatic emission at $6300 \AA$ (as indicated by additional measurements of $5577 \AA$ ( $0\left({ }^{\top} S\right)$ ) and $4278 \AA(N 2+(1 N G))$ emissions) as we11 as the morphology of the subauroral emission. The LAPI instrument consists of 15 electron and 15 ion sensors mounted on a single axis, magnetically oriented scan platform, and provides simultaneous energy and pitch angle information for energies from $5 \mathrm{eV}$ to $32 \mathrm{keV}$. DE-2 was placed in a polar orbit in August 1981. A very important feature of the LAPI is the ability to measure charged particles in discrete energy ranges rather than an energy integrating fashion. During the observations reported here, the altitude of $D E-2$ varied from $295 \mathrm{~km}$ to $950 \mathrm{~km}$ over the ground stations, with the majority of passes in the higher altitude range.

Because our current knowledge of SAR arcs is derived from the relatively few occurrences which have been studied, the task was undertaken to examine a17 SAR arc features that have been detected by the MASP network during the lifetime of the LAPI instrument. This led to the identification of approximately 100 events that were classified as SAR arcs. Using available LAPI measurements, it was found that coincident data existed for 23 passages of $D E-2$ within or near SAR arc regions being viewed by the photometers. An example of these data is shown in Figure 1, showing three cases--two during 21 October 1981, and one during 23 October 1981. Profiles of emission rates at $6300 \AA$ along the satellite paths for these three occasions are shown, together with measurements of electrons $\left(7.5^{\circ}\right.$ pitch angle) from four low energy channels of LAPI. Emission profiles were determined with the assumption that emissions predominant7y originated from $425 \mathrm{~km}$ altitude. Enhanced emission rates associated with the SAR arcs clearly track energy flux being carried downward by low energy electrons within the energy range $5.1 \mathrm{eV}$ to $8.8 \mathrm{eV}$. Equally evident are the simultaneous reductions of electron fluxes at $20.0 \mathrm{eV}$, by as much as an order of magnitude. We interpret the latter feature to be photoelectrons originating in the southern hemisphere.
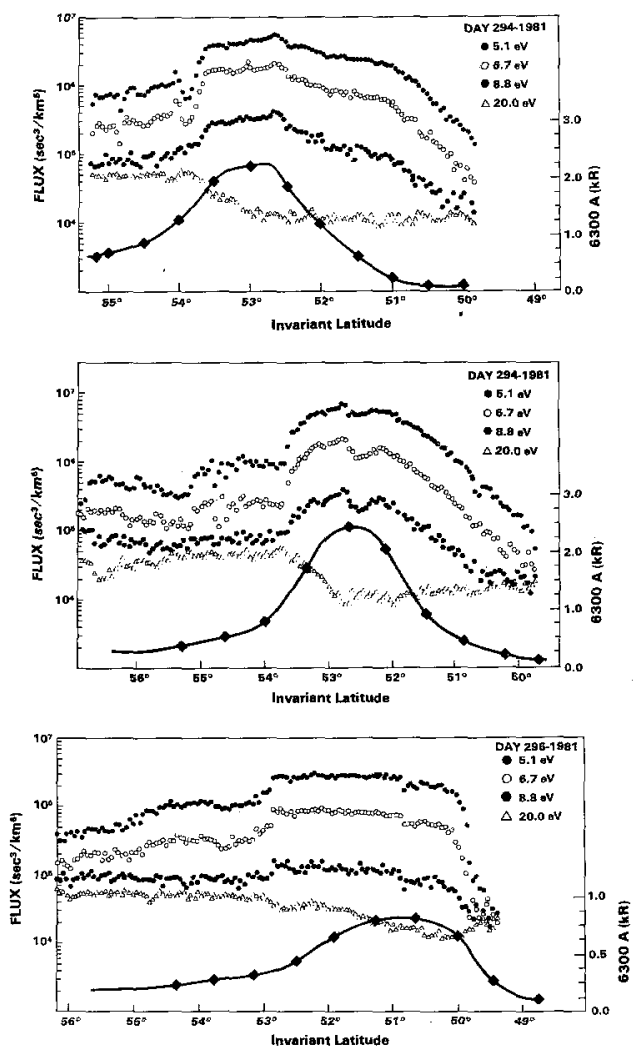

* Fig. 1. $6300 \AA$ emission (MASP) vs. electron fluxes (LAPI). LAPI electron fluxes are 1 second averages; MASP measurements are identified by solid lines.

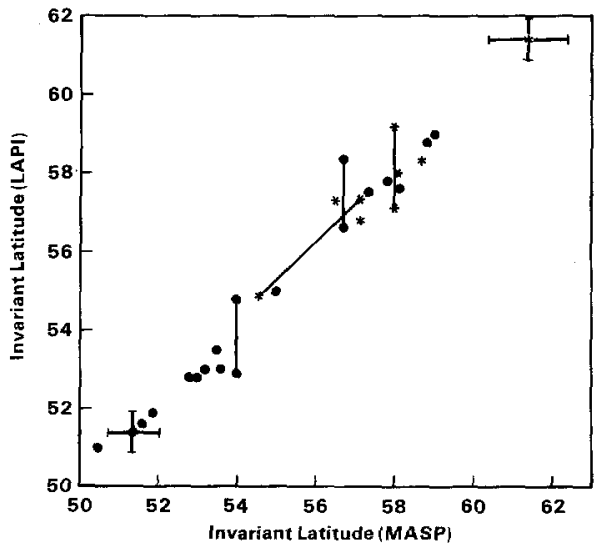

†Fig. 2. Latitudes of SAR arcs.

To establish whether these plasma properties are characteristic of regions conjugate to SAR arc regions, spectrograms were produced for each of the 23 occasions of coincidence or near coincidence. In a 17 cases determined by the photometer network to be SAR arc occurrences, entanced fluxes of low energy electrons were noted by LAPI. The results are plotted in Figure 2, showing the latitude of maximum $6300 \AA$ emission as determined from photometer data as a function of the locations of peak suprathermal electron fluxes $(E<12 \mathrm{eV})$. Those correspondences requiring a slight longitudinal extrapolation $\left(<10^{\circ}\right)$ are depicted by asterisks; features appearing to be double SAR arcs are shown by connected location points. Three of four double SAR arcs (as determined by LAPI) appear as singular structures to the 
MASP instruments. The photometrically determined arc locations for these three instances lie at the center of the line segments joining the particle signatures. Also in Figure 2 are approximate error bars for the Tatitude determinations; potential errors brought about by the limited number of elevation scans performed by the photometers as well as uncertainty of the height distribution of $6300 \AA$ emissions, and the width of particle signatures noted by LAPI. There have been no cases discovered in this study in which an arc has been detected photometrically yet lacked the accompanying low energy electron signature when DE-2 was within the area monitored from the earth.

In the single report describing the presence of precipitating suprathermal electrons on field lines spatially coincident with a monitored 'normal' SAR arc $/ 6 /$ it was demonstrated that the electrons exhibited a downward bulk flow velocity, along field lines threading the arc. Because this may represent an important form of energy transport often overlooked in studies that rely solely on solving heat conduction equations, a broader examination was conducted. Using a procedure similar to that described by Gurgiolo et a 7. 16/, electron distribution function contours were constructed from LAPI measurements for cases depicted in Figure 2. For those cases examined, contours representing precipitating electrons were quite well described by circular arcs offset along the v-parallel axis, thus suggesting the possibility of a Maxwellian description for the fluxes. Offsets of these contours in velocity space were used to estimate field-aligned flow velocities for the electrons associated with the arcs. Typical values for these events appear to 1 ie in the $200 \mathrm{~km} / \mathrm{sec}$ to $300 \mathrm{~km} / \mathrm{sec}$ range. Using these values, and taking into account spacecraft charging determined for each period, the distributions at sma11 pitch angles were transformed to the plasma rest frame. The linearity of the resulting distribution functions immediately suggested that the lower energy electrons associated with the SAR arcs may be described by a Maxwellian distribution. This finding was used for modeling of the events.

\section{MODELING}

To determine if the precipitating electron fluxes associated with SAR arcs could be responsible for the underlying electron temperature enhancement and consequent $6300 \AA$ emissions, calculations of emission profiles within the body of several SAR arcs were made. Three cases were selected; two during 21 0ctober 1981, and one during 23 October 1981 (Figure 1). For each event modeled, an appropriate MSIS model atmosphere was used (atmospheric composition was not measured by DE-2 due to the high altitude of the spacecraft). Estimates of the electron densities up to the F-layer peak were obtained from the Wallops Island or Boulder ionosonde, whichever was closer to the satellite position. Longitudinal positions of the satellite during periods depicted in Figure 1 were $283^{\circ}, 259^{\circ}$, and $272^{\circ}$, respectively. Electron densities were extrapolated within the altitude range above the peak to the value measured at the satelite position by a Langmuir probe. The computed magnitude of the energy carried by the precipitating Maxwellian flux extrapolated to low energies is dependent on what is assumed for the spacecraft potential. Spacecraft potentials for the cases investigated were derived from the Ion Drift Meter onboard DE-2, and were taken to be $1.28 \mathrm{~V}, 1.50 \mathrm{~V}$, and $1.10 \mathrm{~V}$, respectively.

These precipitating electron fluxes were supplied as an upper boundary condition to the twostream electron transport code of Nagy and Banks /7/ for calculation of electron heating rates. For Case 1 (21 0ctober 1981, 01:55:16 UT), fluxes were supplied both with and without the low energy extrapolation for comparison purposes. For a 11 other cases, measured precipitating fluxes along with the low energy extrapolation were used. These rates were then supplied to the truncated version of a code originally developed by Young et a1. /8/ which solves the coupled continuity, momentum and energy equations. The calculations carried out for this modeling required only a solution of the energy equation because the electron density profile was held fixed and heating rates were supplied. The truncated version of this code no longer solves along an entire flux tube but has an upper boundary at the satellite altitude and accepts an additional heat flux as an upper boundary condition if necessary to duplicate electron temperatures measured at the satellite. This heat flux was assumed to be partially the result of heating of the electron gas above the satellite altitude as a resuit of the precipitating electron flux when the kinetic approach at low energies was used. It would represent the entire energy transport if a classical heat flux approach had been used. For the cases employing extrapolation of the precipitating electron fluxes to lower energies, the additional heat flux at the satellite altitude needed to produce the observed electron temperatures was smal1 in comparison to the column heating resulting from the precipitating flux distributions, amounting to $19 \%, 1 \%$, and $26 \%$ of the total energy for Cases 1, 2, and 3, respectively. In these cases, the bulk of the required energy was carried by precipitating electron fluxes, within the uncertainties introduced by the low energy extrapolation. When no low energy extrapolation of the precipitating electron flux was used in Case 1, onty $23 \%$ of the energy was supplied by the measured electron fluxes, the rest being carried by a classical heat flux added as a boundary condition in the calculations.

The question now arises as to whether the calculated temperature profiles are capable of producing the $6300 \AA$ emissions as measured at these times and locations. Column integrated 
quantities derived for the modeled events corresponding to cases 1, 2, and 3 are given in Table 1. Emission rate values within the SAR arc, determined by MASP measurements near the projected $D E-2$ orbital path, are included for comparison. Agreement between measured and computed $6300 \AA$ emission rates are quite good. Note that very little of the $6300 \AA$ emission results from direct collisional excitation by the original precipitating electrons; most is produced by the action of the heated ionospheric electron gas.

IABLE $1 \quad$ Column Integrated Quantities

$\begin{array}{lcccc} & \begin{array}{c}\text { Case 1 } \\ 81294(01: 55: 16 \text { UT) } \\ \text { With Low Energy } \\ \text { Fiux Extrapolation }\end{array} & \begin{array}{c}\text { Case } 1 \\ 81294(01: 55: 16 \text { UT) } \\ \text { Without Low Energy } \\ \text { Ffux Extrapolation }\end{array} & \begin{array}{c}\text { Case } 2 \\ \text { Case } 3\end{array} & \begin{array}{c}\text { (1294 (03:31:56 UT) } \\ 81296 \text { (02:29:51 UT) }\end{array} \\ \text { 6300A Emission Dus to Thermal Electrons (R) } & 2.35 \times 10^{3} & 1.59 \times 10^{3} & 3.26 \times 10^{3} & 8.07 \times 10^{2} \\ \text { 6300A Emission as Measured by MASP Network (R) } & 2.40 \times 10^{3} & 2.40 \times 10^{3} & 2.50 \times 10^{3} & 8.00 \times 10^{2} \\ \text { 6300A Emission Due to Preclpitating Flux (R) } & 95.2 & 80.6 & 80.3 & 61.8\end{array}$

\section{DISCUSSION}

There were several uncertainties encountered in the calculation of an ionospheric response which prevent a unique solution to the energy deposition problem as posed. Of largest potential influence is a lack of direct measurements of the precipitating electron energy distribution below approximately $5 \mathrm{eV}$. For the cases modeled here, typical spacecraft altitudes were approximately $880 \mathrm{~km}$. Consequently, the electron fluxes have already been degraded somewhat by interactions with the atmosphere and ionosphere, with associated heating of the ambient electron gas. Information on the possible form of the distribution functions is available, however. The nature of the measured distribution functions indicates that the electron fluxes observed precipitating over SAR arcs can be approximated as flowing Maxwellians with some specified field-aligned flow velocity and temperature after taking spacecraft potential into account, a procedure used here. Much better model constraints would be provided by measurements at high altitudes by particle detectors capable of examining the electron distribution function at energies below $5 \mathrm{eV}$.

To investigate uncertainties of the calculated $6300 \AA$ emission rates generated by variations of the assumed neutral atmospheric composition, the MSIS exospheric temperature was adjusted to $200^{\circ}$ less and $200^{\circ}$ more than expected for geomagnetic conditions experienced during the cases modeled in this report $\left(1078^{\circ}\right.$ and $1478^{\circ}$ versus $\left.1278^{\circ} \mathrm{K}\right)$. The volume emission rates for $6300 \AA$ as a result of this scaling differed from those derived earlier by only about $10 \%$ with higher temperatures corresponding to higher emission rates.

For sufficiently low energy electron fluxes, any distinction from classical electron heat conduction reduces to semantics. However, if a significant portion of the energy is deposited as heat at altitudes approaching that from which SAR arc emissions originate, this supply of energy must be considered during calculations in addition to the customary heat conduction through the electron gas. We therefore believe that it is important to include in any theoretical framework the presence of energy flux not only in the form of electron gas heat conduction but also of the component indicated by this report, i.e., a heated, convecting population of electrons. These observations pose the question: why have many earlier investigations reported an absence of electron fluxes associated with SAR arcs? Two reasons may be suggested: (1) most attempts have used integrating suprathermal electron detectors, thus leading to the situation where a reduced flux of conjugate photoelectrons may approximately balance the increased measurable electron flux at lower energies that is associated with the SAR arc, and (2) detectors that have a sensitivity threshold greater than $10 \mathrm{eV}$ could easily fail to identify the electrons entirely. Either circumstance would lead to a negative result.

\section{CONCLUSION}

A thorough search of data available at the time of this writing has yielded 23 instances of nearly coincident data from LAPI and the MASP network, although there is clear evidence of hundreds of additional measurements which were not coincident. For every case in which a SAR arc was observed, downward fluxes of low energy electrons ( $E<10$ eV) were enhanced relative to measurements both north and south of the arc. It appears that these electron fluxes, within the constraints imposed by uncertainties of the extrapolations indicated, have sufficient energy to establish the required temperatures and that resulting temperature profiles produce emissions comparable to those observed. It is not yet clear where the electrons reported here originate, whether from relatively low altitudes (perhaps from a heated electron gas within several thousand kilometers of the satellite) or from regions much nearer the equatorial plane. Efforts to determine this are continuing, using measurements by the High Altitude Plasma Instrument (HAPI) in conjunction with LAPI and the MASP network. 


\section{ACKNOWLEDGEMENTS}

The authors' thanks go to L. Brace and W. Hoegy for supplying electron temperature and density information, R. Heelis for determining spacecraft potential, and M. Ertle for determining bottomside electron density profiles. This work was supported by DOE contract DE-AC06-76RLO-1830, and NASA under grants NAG5-472 and NGR-23-005-015, and NSF under grant ATM8508753.

\section{REFERENCES}

1. S. Chandra, E.J. Maier, B.E. Troy, Jr., and B.C. Narasinga Rao, Subauroral Red Arcs and Associated ionospheric Phenomena, J. Geophys, Res. 80, 920 (1971)

2. A.F. Nagy, L, H. Brace, N.C. Maynard, and W.B. Hanson, Is the Red Arc a Good Indicator of Ionospheric-Magnetospheric Conditions?, J. Geophys. Res. 79, 4331 (1974)

3. E.J. Maier, S. Chandra, L. Brace, J.H. Hoffman, G.G. Shepherd, and J.H. Whitteker, The SAR Red Arc Event Observed During the December 1971 Magnetic Storm, J. Geophys. Res. $75,6260(1975)$

4. J.S. Prasad, J.S. Kim, and S. Okano, Observations of Soft Electron Flux During SAR ArC Event, Plant. Space Sci. 28, 375 (1980)

5. G.G. Shepherd, L.H. Brace, J.R. Burrows, J.H. Hoffman, H.G. James, D.M. Klumpar, A.F. Nagy, E. Stathopoulos, and J.H. Whitteker, An Unusual SAR Arc Observed During Ring Current Development, 4 August 1972, Plant. Space Sci. 28, 69 (1980)

6. C. Gurgiolo, D.W. Slater, J.D. Winningham, and J.L. Burch, Observation of a Heated Electron Population Associated with the 6300A SAR Arc Emission, Geophys. Res. Lett. 9, $965(1982)$

7. A.F. Nagy and P.M. Banks, Photoelectron Fluxes in the Ionosphere, J. Geophys. Res, 75, $6260(1970)$

8. E.R. Young, D.G. Torr, P. Richards, and A.F. Nagy, A Computer Simulation of the Mid latitude Plasmasphere and Ionosphere, Planet. Space Sci. 28, 881 (1980) 